\title{
Transitando de Embrión a Feto: La Metamorfosis de los Cordados
}

\author{
Transiting from Embryo to Fetus: The Metamorphosis of the Chordata
}

\author{
Carolina Smok $^{1,2}$; Ignacio Roa ${ }^{3,4,5}$; Ruth Prieto ${ }^{6}$ \& Mariana Rojas ${ }^{2}$
}

SMOK, C.; ROA, I.; PRIETO, R. \& ROJAS, M. Transitando de embrión a feto: la metamorfosis de los cordados. Int. J. Morphol., 36(2):709-715, 2018.

RESUMEN: Durante el período del desarrollo conocido como prefetal, el embrión cambia sus características ictiomórficas comunes a todos los vertebrados y adquiere gradualmente las formas propias de la especie que representa. Durante este período se forma la cara, involucionan los arcos faríngeos (branquiales) formándose el cuello, y aparecen los miembros. Se constituye, además, la hernia umbilical fisiológica, que consiste en la presencia de asas intestinales dentro del cordón umbilical. El sistema nervioso origina las vesículas telencefálicas, el diencéfalo, mesencéfalo, metencéfalo, y mielencéfalo. Este periodo corresponde a una etapa de máxima susceptibilidad ante los teratógenos que pueden generar malformaciones en todas las especies animales. El objetivo del presente trabajo es presentar los principales eventos acontecidos durante el periodo prefetal, además de una visión y opinión de los autores, proponiendo una nueva denominación a la etapa: periodo metamórfico.

PALABRAS CLAVE: Embrión; Prefetal; Organogénesis; Período metamórfico.

\section{INTRODUCCIÓN}

Durante el estado filotípico de los embriones (en el cual todos los vertebrados son similares), es difícil reconocer la especie a la cual pertenecen (Wolpert, 2009). A medida que avanzan en el desarrollo, estos experimentan profundos cambios involucrandose forma y función. El término internacionalmente utilizado para este periodo es prefetal, extendiéndose desde los 36 a 56 días; otro término utilizado es el de organogénesis. Según O’Rahilly \& Müller (2001) en la especie humana este período corresponde a las etapas Carnegie 13-23. Por otra parte el nombre metamórfico (del griego $\mu \varepsilon \tau \alpha$ (meta, 'cambio') y $\mu о \rho \phi \eta$ (morph, 'forma'), ha sido propuesto con el fin de explicar la serie de cambios de forma que sufre el embrión durante su desarrollo y hacen posible reconocer su especie.

Este periodo es considerado esencial, crítico durante el desarrollo y organogénesis, debido a que en esta etapa continúa la diferenciación de los órganos que comenzaron a formarse durante el periodo somítico, y además se forman nuevos esbozos, y por ello puede ser alterada por agentes teratogénicos (Rojas \& Walker, 2012). En la especie humana se sabe que el alcohol y nicotina, drogas como la cocaína, fármacos como anticonceptivos orales que contienen progestágenos y estrógenos, ácido retinoico en grandes dosis, cafeína, alimentos o agua contaminada con residuos industriales como mercurio orgánico o bifenilos policlorinados pueden actuar como agentes teratogénicos (Holmes, 2011).

El objetivo del presente trabajo es presentar los principales eventos acontecidos durante el periodo prefetal, además de una visión y opinión de los autores, proponiendo una nueva denominación a la etapa: período metamórfico.

Principales Eventos del Período Prefetal o Metamórfico. Los embriones de los cordados empiezan a diferenciar sus características externas después del período somítico o filotípico. Algunos de los caracteres que nos permiten reconocer la especie corresponden a los miembros y la cara.

\footnotetext{
${ }^{1}$ Instituto I3S, Facultad de Ciencias de la Salud, Universidad Central de Chile, Chile.

${ }^{2}$ Laboratorio Embriología Comparada. Programa de Anatomía y Biología del Desarrollo, Facultad de Medicina, ICBM. Universidad de Chile, Chile.

${ }^{3}$ Unidad de Morfología, Departamento de Ciencias Básicas Biomédicas, Facultad de Ciencias de la Salud, Universidad de Talca, Talca, Chile.

${ }^{4}$ Programa de Doctorado en Ciencias Morfológicas, Facultad de Medicina, Universidad de La Frontera, Temuco, Chile.

${ }^{5}$ Becario CONICYT-PCHA/Doctorado Nacional/2015-21150235.

${ }^{6}$ Facultad de Medicina, Universidad de La Frontera, Temuco, Chile.
} 

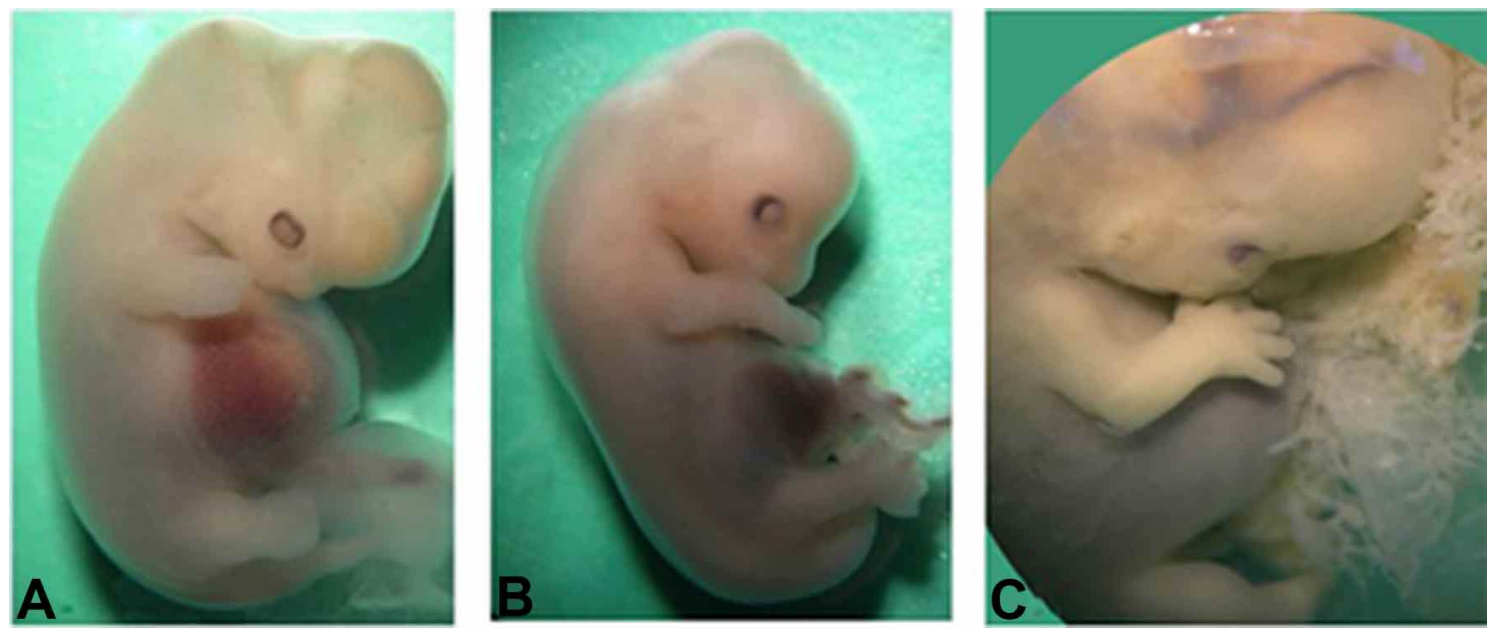

Fig. 1. Embriones de mamíferos en etapa prefetal. 1A: embrión bovino (Bos taurus) de $20 \mathrm{~mm}$. El cuerpo es curvado, en la cara en formación se visualiza el ojo con pigmento de la retina y la oreja está a nivel del cuello. Los miembros torácicos presentan un surco interdigital, y el miembro posterior carece de estos. 1B: embrión felino (Felis catus) de mayor desarrollo. La cara está prácticamente formada. Los miembros anteriores presentan los tres segmentos, y en los miembros posteriores se observan 4 surcos interdigitales delimitando los cinco dedos, existiendo membranas interdigitales entre ellos. Nótese el cordón umbilical con hernia umbilical fisiológica en su interior. 1C: embrión humano. La cara presenta un avanzado desarrollo, con ojos de posición lateral y orejas con implantación baja. Se observa el miembro superior con los 3 segmentos que lo componen, y se evidencian los 5 dedos de la mano.

En la Figura 1 se observan embriones de distintas especies: bovino (1A), gato (1B), humano (1C). En todos ellos se observan miembros superiores (torácicos) e inferiores (pélvicos), y los arcos faríngeos han involucionado para formar la cara y cuello, además se pueden identificar las vesículas ópticas.

Región Cervico-Craneo-Facial. Durante el período somítico aparecen 3 pares de prominencias faciales dispuestas alrededor de la cavidad oral primitiva o estomodeo: i) prominencia frontonasal, iii) dos prominencias maxilares $\mathrm{y}$, iv) dos prominencias mandibulares (estas 2 últimas derivadas del primer par de arcos faríngeos). La prominencia frontonasal rodea la porción ventrolateral del cerebro anterior, originando posteriormente las placodas ópticas y olfatorias (Van Cruchten et al., 2017). El par de prominencias maxilares diferenciarán los límites laterales del estomodeo; mientras que, las prominencias mandibulares constituirán los límites caudales de la cavidad oral primitiva (Meruane et al., 2012).

Durante el período metamórfico, el mesénquima de los márgenes de las placodas olfatorias prolifera, dando origen a las prominencias nasales medial y lateral. (Fig. 2). Un surco nasolagrimal separa el proceso nasallateral del maxilar del lado correspondiente, mientras que la fisura oronasal separa los procesos maxilares de los nasales mediales (Fig. 3). En la medida que estas prominencias fa- ciales proliferan, se van fusionando entre ellas: la maxilar comienza a unirse con la prominencia nasal lateral, posteriormente se fusionan las prominencias nasales mediales entre sí, y finalmente se fusionan con las maxilares (Fig. 3). Este cierre ocurre a las siete semanas de gestación en el humano, a las 8 semanas en la vaca, 7 semanas en el caballo, 33 días en el canino y en el cerdo, 32 días en el gato y 27 días en el hurón (Noden \& de Lahunta, 2001).

Una semana más tarde las orejas inician su desarrollo alrededor del primer surco faríngeo, donde se forman los colículos auriculares (Fuchs \& Tucker, 2015; Anthwal \& Thompson, 2016).

A medida que se fusionan las prominencias nasales mediales entre sí, éstas conforman el primordio palatino (Fig. 3 ), originando el philtrum labial, el segmento premaxilar de la maxila, incluida la encía y el paladar primario (Fig. 3A y B). A partir de las prominencias maxilares se forman: las porciones laterales del labio superior, la mayor parte del maxilar y los procesos palatinos secundarios (crestas palatinas) (Fig. 3B y C). Los procesos nasolaterales desarrollan alas de la nariz. Los labios y mejillas primitivos son invadidos por mesénquima proveniente del segundo arco faríngeo, el cual diferenciará los músculos faciales. Estos músculos de la expresión facial recibirán su inervación del nervio del segundo arco, nervio facial (Meruane et al.; Som \& Naidich, 2013, 2014). 


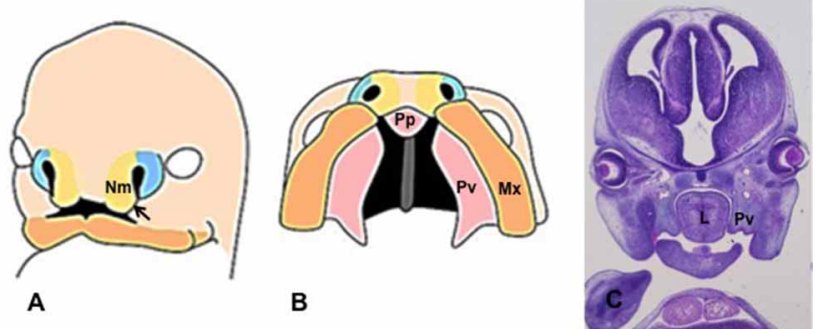

Fig. 2. Formación de la cara durante la sexta semana del desarrollo humano. Se observan las fositas olfatorias y las prominencias nasales mediales (amarillo) y las nasoles laterales (celeste). 2A: La fisura oronasal separa los procesos maxilares de los nasales mediales (Nm) (flecha). 2B: Se observa el paladar primario (Pp), desde los procesos maxilares $(\mathrm{Mx})$ crecen los procesos palatinos laterales en sentido vertical (Pv). 2C. Se observa la lengua (L) y a ambos lados las prominencias palatinas verticales $(\mathrm{Pv})$.
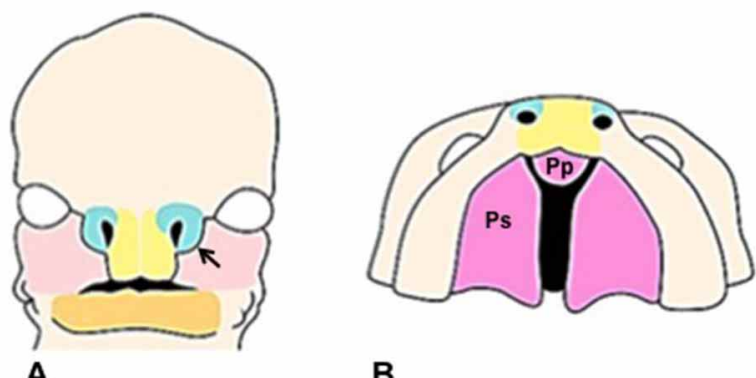

Fig. 3. Formación de la cara durante la séptima semana. 3A: surco nasolagrimal (flecha), prominencia nasal medial (amarilla), prominencia nasal lateral (celeste), prominencia maxilar (rosado), prominencia mandibular (naranjo). 3B: paladar primario $(\mathrm{Pp})$, procesos palatinos secundarios (Ps).

Durante este periodo es posible distinguir las diferencias de la cara de bovinos, cerdos, equinos, y algunas razas caninas, en relación a felinos y humanos, ya que en los primeros se genera un crecimiento acelerado de las prominencias nasales, maxilares y mandibulares (Figs. 6 y 10).

Los arcos faríngeos experimentan cambios importantes: a partir del segundo arco faríngeo existe un crecimiento del margen caudal llamado opérculo, el cual cubre los restantes arcos, constituyéndose el seno cervical (Fig. 4), el cual posteriormente oblitera debido al crecimiento y fusión de sus paredes, dando origen al cuello liso (Figs. 1, 6 y 10). Las células mesenquimales de los arcos faríngeos dan origen a los huesos de la cara, cartílagos laríngeos y los oscículos del oído medio. Estas mismas células dan origen a la musculatura facial, masticatoria y a los componentes conectivos de la lengua. Sin embargo, los componentes epiteliales de las glándulas tiroides y paratiroides, y de los órganos linfoides, timo y tonsilas se diferencian a partir del endodermo de la faringe (Meruane et al.)

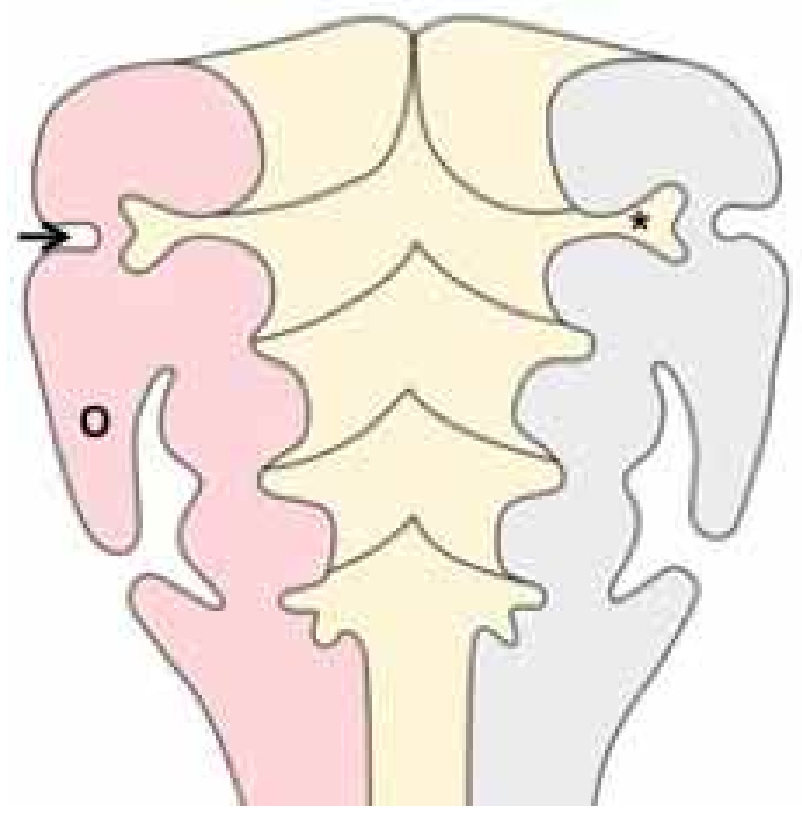

Fig. 4. Corte de arcos faríngeos. Desde el primer surco faríngeo se forma el conducto auditivo externo (flecha). La primera bolsa faríngea forma el receso tubo-timpánico (*). El segundo arco crece hacia caudal para formar el opérculo $(\mathrm{O})$, el cual prolifera para cubrir los surcos $2^{\mathrm{a}}, 3^{\circ}$ y $4^{\circ}$ formando el seno cervical.

\section{Diferenciación del Sistema Nervioso.}

En el embrión prefetal, las vesículas encefálicas sufren una serie de modificaciones: la vesícula prosencefálica (cerebro anterior) da origen a dos dilataciones laterales llamadas vesículas telencefálicas y al diencefálo de ubicación central (Fig. 5). Posteriormente, la pared de las vesículas telencefálicas se adelgaza y las invaginaciones de la piamadre vascular forman en ese sitio el plexo coroídeo de los ventrículos laterales, a su vez, en el piso de cada vesícula telencefálica se forma el cuerpo estriado (O'Rahilly \& Müller; Müller \& O'Rahilly, 2003; Rojas et al., 2011).

Por otra parte desde la pared del diencéfalo se forman: el epitálamo, el tálamo y el hipotálamo. El tálamo crece con rapidez protruyendo hacia el tercer ventrículo (luz del diencéfalo). El hipotálamo da origen a la estructura adulta del mismo nombre, mientras que el epitálamo da origen a la glándula pineal (Roa \& del Sol, 2014).

El mesencéfalo sufre menos modificaciones que las otras regiones del cerebro. La luz de esta vesícula se estrecha para formar el acueducto cerebral. En el techo del mesencéfalo se desarrollan los colículos superior e inferior, que consisten en cuatro grandes grupos de neuronas, que se 


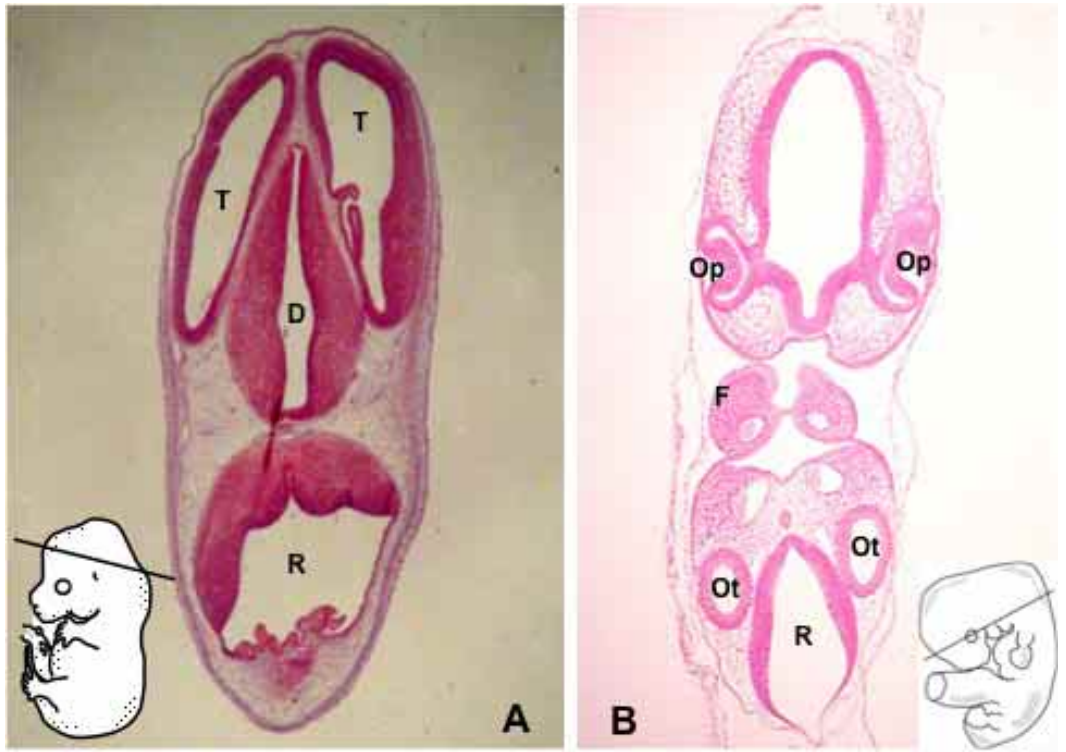

Fig. 5. Corte coronal de de cabeza de embrión. 5A: Vesículas telencefálicas (T), Diencéfalo (D) y Rombencéfalo (R ). Figura 5B: Diencéfalo, Vesículas ópticas (Op), Arcos Faríngeos (F), Romboencéfalo (R), Vesículas óticas (O).
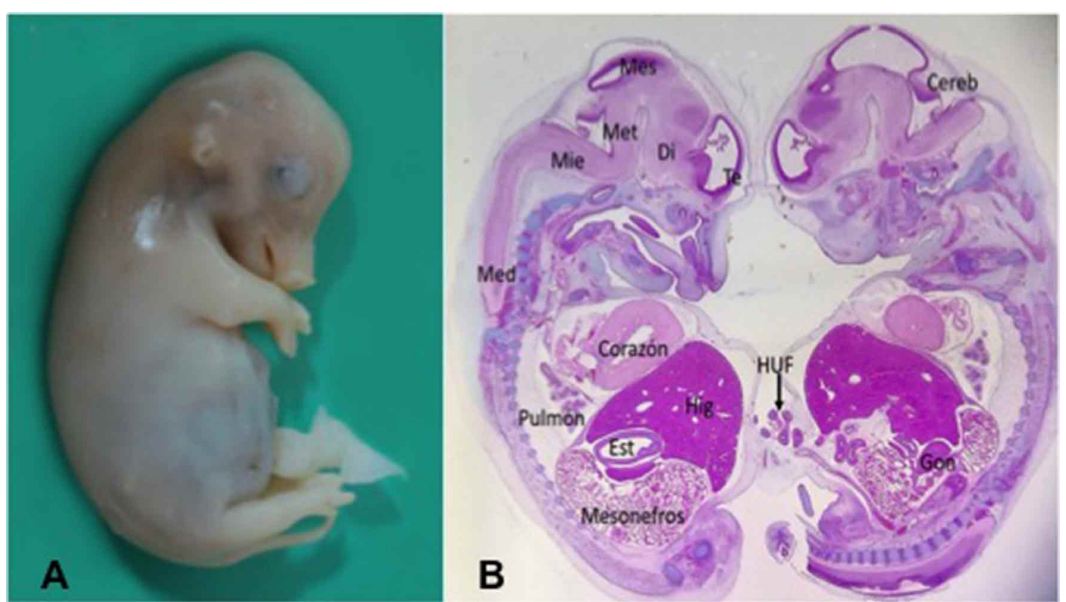

Fig. 6. Embrión de cerdo (Sus scrofa). 6A: embrión in toto. La cara y los miembros están adquiriendo la forma definitiva de la especie. 6B: Corte sagital de embrión de cerdo de $18 \mathrm{~mm}$ LCC. Presencia de hernia umbilical fisiológica (HUF) y esqueleto cartilaginoso. Te (Telencéfalo), Di (Diencéfalo), Mes (Mesencéfalo), Met (Metencéfalo), Mie (Mielencéfalo), Cereb (Cerebelo), Med (Médula), Hig (Hígado), Est (Estómago), Gon (Gónada).

relacionarán posteriormente con los reflejos visuales y auditivos. En la porción basal del mesencéfalo se encuentran fibras que vienen del cerebro en crecimiento formando los pedúnculos cerebrales (Rodriguez et al., 2015).

El rombencéfalo origina el metencéfalo y el mielencéfalo (Fig. 5). Desde el metencéfalo se forma el cerebelo, que actúa como centro de coordinación de la postura y el movimiento, y también la protuberancia, que sirve de vía para las fibras nerviosas que conectan la médula espinal con la corteza cerebral y cerebelar. El mielencéfalo se modifica poco y se convierte en el bulbo raquídeo. La luz del metencéfalo y mielencéfalo corresponde al cuarto ventrículo y, al igual que los ventrículos laterales y el tercer ventrículo, contiene plexos coroideos derivados de una invaginación de la piamadre vascular que se caracterizan por elaborar el líquido cerebroespinal (Rodriguez et al.).

La hipófisis se desarrolla a partir de dos primordios ectodérmicos, al principio separados, que luego se fusionan. Uno de ellos es el infundíbulo que se forma como un crecimiento en sentido ventral del suelo del diencéfalo. El otro primordio es formado del techo del estomodeo y se denomina bolsa adenohipofisiaria (de Rathke). La aposición de estas dos estructuras se mantiene aún cuando con el crecimiento aumenta la distancia entre el suelo del diencéfalo y el techo de la cavidad oronasal. Durante este período la bolsa adenohipofisiaria se cierra, formando una vesícula separada del estomodeo, sus células epiteliales se diferencian en células endocrinas de la adenohipófisis, que forma la pars distalis y pars intermedia de la hipófisis. El infundíbulo crece por la cara caudal de la adenohipófisis y dará origen a la neurohipófisis (Noden \& de Lahunta).

El tubo neural se diferencia en 3 capas: i) ependimaria, ii) del manto (futura sustancia gris) y, iii) velo marginal (futura sustancia blanca). El tubo neural se divide también en una placa alar dorsal y una placa basal ventral, ésta última corresponde al componente motor de la médula espinal, mientras que la placa alar es sensitiva. Además se constituye la placa del techo y la placa del piso (Fig. 7).

Miembros. Al inicio del período prefetal, aparecen en primer lugar los esbozos de los miembros superiores (anteriores en cuadrúpedos); y después los esbozos de los miembros inferiores (posteriores en cuadrúpedos). Sin embargo su desarrollo es similar. Cada esbozo está constituido por tejido mesenquimal del mesodermo lateral somático, y revestido de una capa de células epiteliales ectodérmicas, en cuyo extremo se observa un engrosamiento, denominado cresta ectodérmica apical (CEA) (Fig. 8) (Antonelli et al., 2012; Carlson, 2014; Pineault \& Wellik, 2014). 


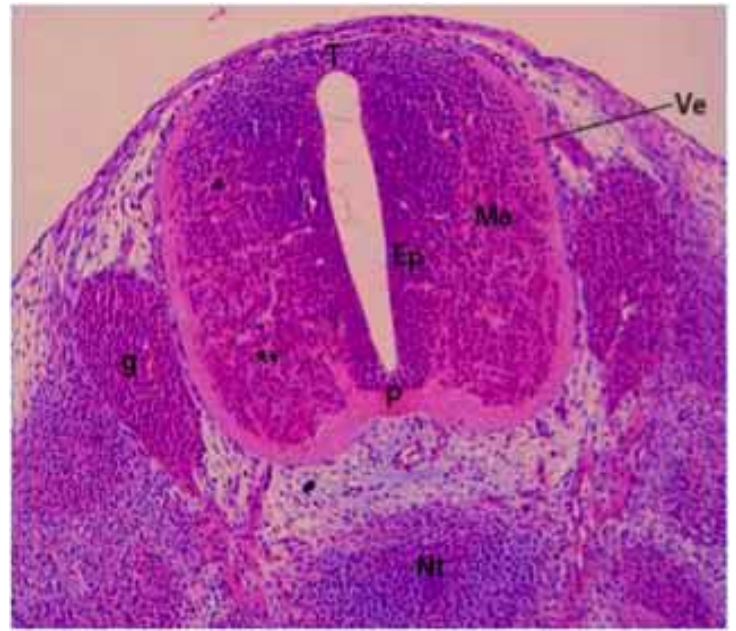

Fig. 7. Corte transversal de tubo neural de embrión. Capa ependimaria (Ep), Capa del manto (Ma), Velo Marginal (Ve), Placa del techo (T), placa del piso (P), ganglio nervioso $(\mathrm{g})$, notocorda $(\mathrm{Nt})$.

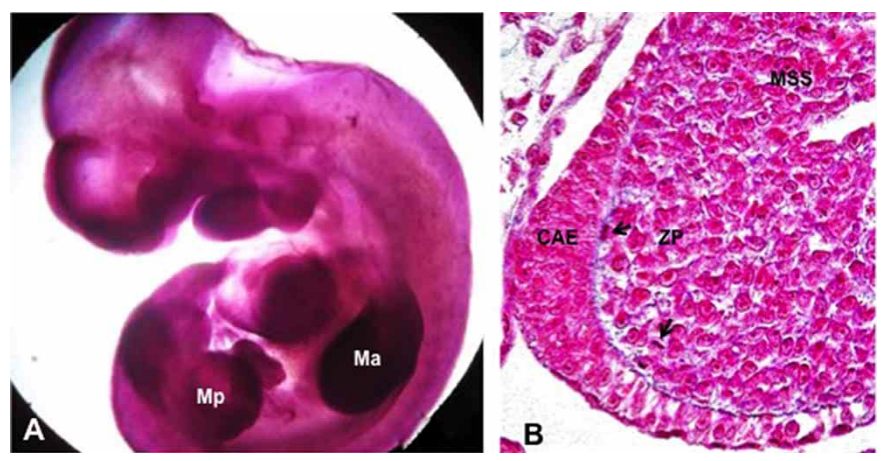

Fig. 8. Embrión del roedor (Octodón degus) in toto. 8A: Se observa esbozo de miembros anterior (Ma) y posterior (Mp), ambos en forma de paleta. 8B: Corte sagital de esbozo de miembro donde se identifica el epitelio de revestimiento constituyendo la cresta ectodérmica apical (CEA), y Mesodermo lateral somático (MSS). Subyacente a la CEA está la Zona de progreso (ZP) caracterizado por múltiples mitosis (ver flechas). Tinción H/E.
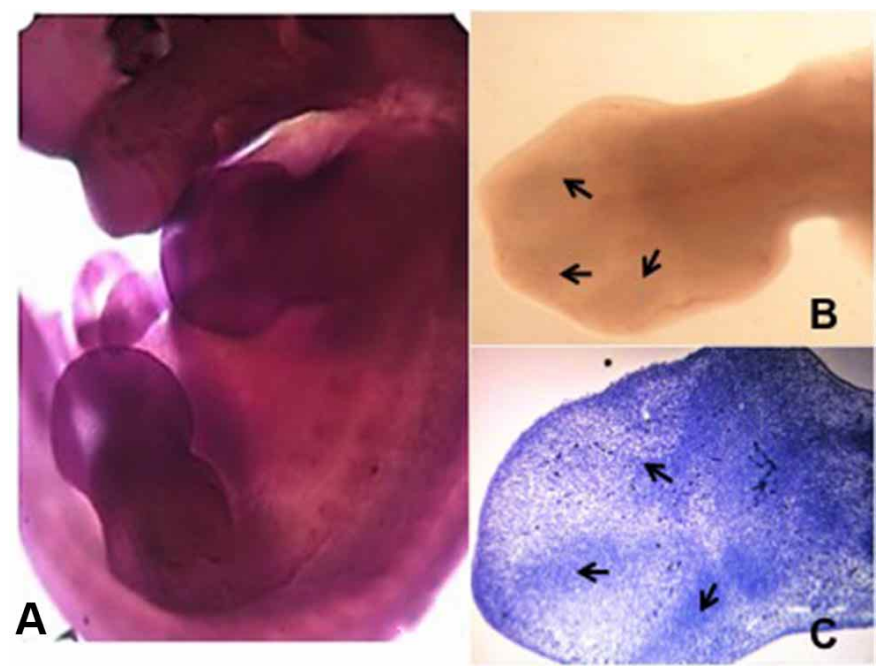

El desarrollo de la CEA es fundamental, ya que ejerce una influencia inductiva en el mesénquima, lo que promueve el crecimiento en longitud del miembro (Zona de progreso) (Carlson). Además de producirse la elongación de este esbozo, aparecen constricciones que delimitan los tres segmentos principales del miembro. En los embriones de mamíferos se observan los futuros dedos en forma de rayos digitales, unidos por una membrana digital. Los miembros de ungulados presentan dos dedos principales, y dos vestigiales (Fig. 9), mientras que felinos, caninos, roedores y humanos presentan cinco dedos. Además, se forman los modelos mesenquimáticos de los huesos de los miembros (Fig. 9C), que posteriormente se diferenciarán a cartílago. Sin embargo la osificación no comienza hasta el periodo fetal (Smok et al., 2014). Finalmente, se produce el alargamiento de los miembros, y los dedos se separan por un proceso de apoptosis de esta membrana interdigital (Antonelli et al.) (Fig. 10).

La histogénesis, que muestra un gradiente próximo-distal muy marcado, es decir, primero se diferencian los tejidos más proximales (del brazo o muslo) y por último los más distales (de la mano o pie). En especies en que se requiere que estos dedos queden libres, este tejido interdigital es eliminado por apoptosis. En relación a su origen, los huesos, ligamentos y vasos sanguíneos de los miembros se forman a partir del mesoderma lateral somático, mientras que el tejido muscular se forma a partir del miotomo del mesoderma somítico.

Sistemas Esplácnicos. En los mamíferos en el período metamórfico o prefetal, la longitud del embrión se mantiene sin variaciones importantes, a diferencia de lo que ocurre con los órganos internos que experimentan un gran desarrollo. El corazón se tabica, El intestino aumenta de longitud y no puede ser contenido dentro de la cavidad abdominal debido al gran desarrollo del mesonefros, de modo que se hernia hacia el exoceloma del cordón umbilical, constituyendo la hernia umbilical fisiológica (Figs. 1 y 6). A principios del periodo fetal las asas intestinales retornan a la cavidad abdominal (Roa \& Meruane, 2012). Durante este período el aparato respiratorio se encuentra en la fase pseudoglandular, es decir se ha desarrollado el sistema

Fig. 9. Embrión de roedor (Octodón degus) en período prefetal. 9A: Embrión in toto. En los miembros anteriores se observan rayos digitales, no así en los posteriores. 9B: Miembro anterior donde se observa por transparencia los modelos mesenquimáticos de los dedos. 9C: Corte de chequeo del mismo miembro anterior. Se observa los rayos interdigitales como condensaciones mesenquimales. Azul de Toluidina, 400X. 

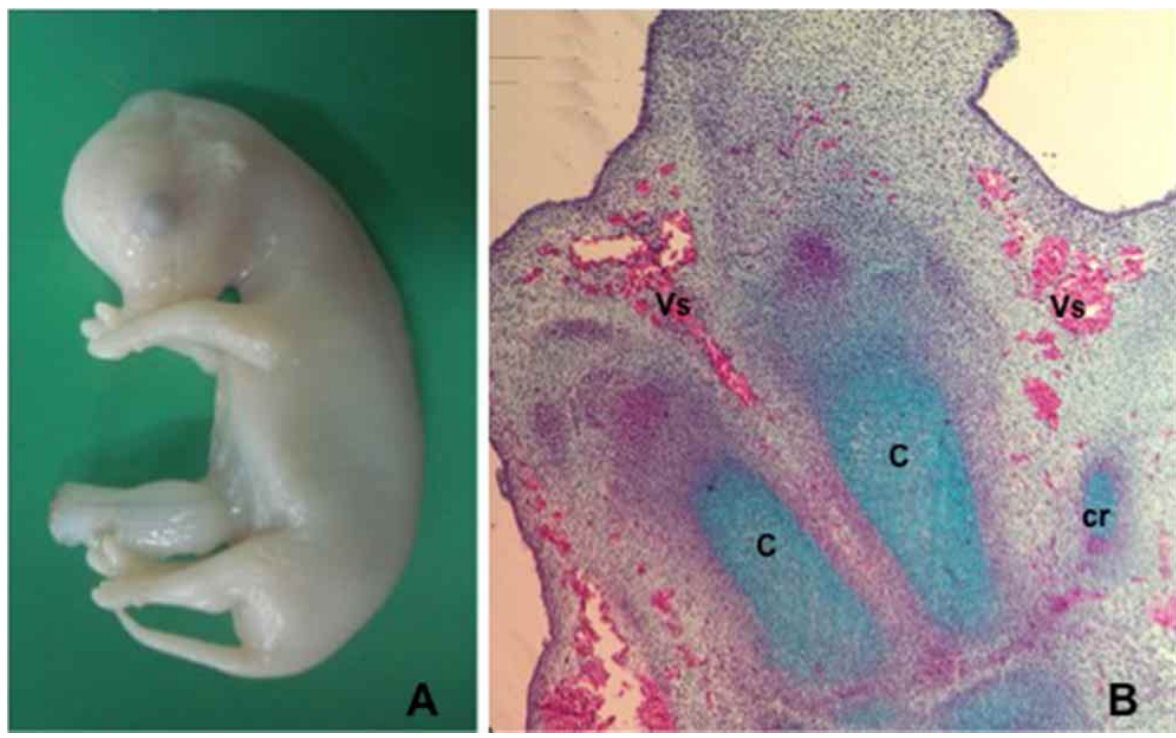

Fig. 10. Embrión de bovino (Bos taurus) en período prefetal. 10A: los miembros presentan dos dedos principales separados por un surco y dos dedos prácticamente vestigiales. 10B: corte histológico del mismo miembro anterior, se observan dos cartílagos centrales $(\mathrm{C})$ que corresponden a los dedos principales y cartílagos reducidos (cr). Existen vasos sanguíneos (Vs) en los lugares donde se encontrarán los surcos interdigitales. H/E-Azul de Alcián, 100X.

de conductos pero no hay presencia de alvéolos, no hay surfactante pulmonar y la vida independiente de la madre es imposible. El sistema urinario ha desarrollado un sistema de riñones intermedios tales como pronefros, mesonefros y metanefros, sin embargo, la función de excreción está a cargo de la placenta (Lemus et al., 2015). Durante este período se forman las gónadas que son indiferenciadas, dos conductos mesonéfricos y dos paramesonéfricos que también son indiferenciados. La diferenciación sexual de las gónadas, genitales externos y diferenciación sexual del sistema nervioso no ocurre hasta el período fetal (Rojas \& Troncoso, 2013; Rojas \& Prieto, 2014).

\section{CONCLUSIÓN}

Como ya hemos visto, durante la etapa prefetal, acontecen cambios significativos en el embrión, que lo acercan cada vez más al aspecto propio de su especie. Es durante esta etapa donde tanto estructuras de cabeza y cuello, miembros y sistémicas son conformadas y comienzan su desarrollo y son más susceptibles a los agentes teratógenos. El paulatino cambio de forma del embrión es lo que nos lleva a plantear que el nombre más apropiado para esta etapa sería la de metamórfica, nombre más explicativo y fácil de relacionar, tanto para estudiantes como académicos. El término metamórfico además se encuentra en la línea a lo expresado por la International Federation of Associations of Anatomists (IFAA), en que los términos se denominen con una sola palabra, el lenguaje empleado sea el latín, se describa el elemento, concentrando la información y descripción (FIPAT, 2013).
Sin lugar a dudas el estudio de la embriología juega un rol preponderante dentro de las Ciencias Morfológicas, por lo cual la correcta denominación de los segmentos, así como los procesos y etapas es esencial; por lo cual la utilización de términos que sean sencillos de comprender y relacionar facilitaría aún más su entendimiento.

SMOK, C.; ROA, I.; PRIETO, R. \& ROJAS, M. Transiting from embryo to fetus: the metamorphosis of the chordata. Int. J. Morphol., 36(2):709-715, 2018.

SUMMARY: During the period of development known as prefetal, the embryo changes its ictiomorphic characteristics common to all vertebrates and gradually acquires the proper forms of the species it represents. During this period the face is formed, the pharyngeal arches (branchial) involute forming the neck, and the limbs appear. In addition, the physiological umbilical hernia is constituted, which consists of the presence of intestinal loops inside the umbilical cord. The nervous system originates the telencephalic vesicles, the diencephalon, mesencephalon, metencephalon, and myelencephalon. This period corresponds to a stage of maximum susceptibility to teratogens that can generate malformations in all animal species. The objective of this paper is to present the main events that took place during the preferential period, as well as a vision and opinion of the authors, proposing a new name for the stage: metamorphic period.

KEY WORDS: Embryo; Prefetal; Organogenesis.

\section{REFERENCIAS BIBLIOGRÁFICAS}

Anthwal, N. \& Thompson, H. The development of the mammalian outer and middle ear. J. Anat., 228(2):217-32, 2016. 
Antonelli, M.; Rosas, C. \& Rojas, M. Limb development in vertebrates. Int. J. Morphol., 30(4):1512-9, 2012.

Carlson, B. Embriología Humana y Biología del Desarrollo. $5^{\text {a }}$ ed. Madrid, Elsevier, 2014.

Federative International Programme for Anatomical Terminology (FIPAT). Terminologia Embryologica. Stuttgart, Thieme, 2013.

Fuchs, J. C. \& Tucker, A. S. Development and integration of the ear. Curr. Top. Dev. Biol., 115:213-32, 2015.

Holmes, L. B. Human teratogens: update 2010. Birth Defects Res. A Clin. Mol. Teratol., 91(1):1-7, 2011.

Lemus, L.; Fuenzalida, M. \& Rosas, C. Desarrollo del aparato urinario. Int. J. Med. Surg. Sci., 2(2):447-54, 2015.

Meruane, M.; Smok, C. \& Rojas, M. Face and neck development in vertebrates. Int. J. Morphol., 30(4):1373-88, 2012.

Müller, F. \& O'Rahilly, R. Segmentation in staged human embryos: the occipitocervical region revisited. J. Anat,. 203(3):297-315, 2003.

Noden, D. \& de Lahunta, A. Embriología de los Animales Domésticos. Zaragoza, Acribia S. A., 2001.

O’Rahilly, R. \& Müller, F. Human Embryology \& Teratology. $3^{\text {rd }}$ ed. New York, Wiley-Liss, 2001.

Pineault, K. M. \& Wellik, D. M. Hox genes and limb musculoskeletal development. Curr. Osteoporos. Rep., 12(4):420-7, 2014.

Roa, I. \& del Sol, M. Pineal gland morphology - A literature review. Int. J. Morphol., 32(2):515-21, 2014.

Roa, I. \& Meruane, M. Digestive system development. Int. J. Morphol., 30(4):1285-94, 2012.

Rodriguez, A. R.; Dominguez, S.; Cantin, M. \& Rojas, M. Embriología del sistema nervioso. Int. J. Med. Surg. Sci., 2(1):385-400, 2015.

Rojas, M \& Prieto, R. Embriología del Aparato Genital de la Mujer. En: Pérez Sanchez, A. \& Donoso-Siña, E. (Eds.). Ginecología. $4^{\text {a }}$ ed. Santiago de Chile, Mediterráneo, 2014.

Rojas, M \& Troncoso, P. Desarrollo Embrionario y Fetal del Aparato Genital Femenino. En: Paris, E.; Sánchez, I.; Beltramino, D. \& Copto, A. (Eds.). Meneghello: Tratado de Pediatría. $6^{a}$ ed. Buenos Aires, Médica Panamericana, 2013.

Rojas, M. \& Walker, L. Congenital malformations: General and genetic aspects. Int. J. Morphol., 30(4):1256-65, 2012.

Rojas, M.; Rodríguez, A. \& Montenegro, M. A. Desarrollo Embrionario y Elementos de Fetación. En: Pérez Sánchez, A. \& Donoso Siña, E. (Eds.). Obstetricia. $4^{\mathrm{a}}$ ed. Santiago de Chile, Mediterráneo, 2011. pp.108-36.

Smok, C.; Roa, I. \& Rojas, M. Desarrollo fetal en mamíferos. Int. J. Med. Surg. Sci., 1(2):139-45, 2014.

Som, P. M. \& Naidich, T. P. Illustrated review of the embryology and development of the facial region, part 1: Early face and lateral nasal cavities. A. J. N. R. Am. J. Neuroradiol., 34(12):2233-40, 2013.

Som, P. M. \& Naidich, T. P. Illustrated review of the embryology and development of the facial region, part 2: Late development of the fetal face and changes in the face from the newborn to adulthood. A. J. N.R. Am. J. Neuroradiol., 35(1):10-8, 2014.

Van Cruchten, S.; Vrolyk, V.; Perron Lepage, M. F.; Baudon, M.; Voute, H.; Schoofs, S.; Haruna, J.; Benoit-Biancamano, M. O.; Ruot, B. \& Allegaert, K. Pre- and postnatal development of the eye: A species comparison. Birth Defects Res., 109(19):1540-67, 2017.

Wolpert, L. Principios del Desarrollo. $3^{\mathrm{a}}$ ed. Madrid, Médica Panamericana, Buenos Aires, 2009.
Dirección para correspondencia:

Dra. Mariana Rojas R.

Laboratorio de Embriología Comparada

Programa de Anatomía y Biología del Desarrollo

Facultad de Medicina, ICBM

Universidad de Chile

CHILE

E-mail: dramrojas@hotmail.com

Recibido : 04-01-2018

Aceptado: 26-03-2018 